\title{
EVOLUTION, GENES, AND BEHAVIOR
}

\author{
by Ian Tattersall
}

\begin{abstract}
The pseudoscience of evolutionary psychology purports to explain human behaviors by reference to an ancestral environment (essentially, a hunting-gathering way of life) in which we evolved. Contemporary human behaviors are allegedly governed directly by genes that reflect adaptation to this environment by natural selection. However, the evolutionary process is much more complex than this reductionist approach implies, and adaptation cannot involve the fine-tuning of structures or behaviors within individuals or species: natural selection can only affect entire organisms, not their components. Similarly, genetic processes are too complex to admit this simplistic view. Instead, our flexible, complex human behaviors probably represent an emergent acquisition.
\end{abstract}

Keywords: evolution; evolutionary process; evolutionary psychology; human behavior; human consciousness; selfish genes; sociobiology.

It has lately become fashionable to "explain" many, if not all, aspects of our frequently unfathomable modern human behaviors by reference to "the genes," or, more indirectly, to the "ancestral environment" in which those genes and their associated behavior patterns allegedly evolved (e.g., Wright 1998). This unfortunate development has occurred under the umbrella of the pseudoscience of evolutionary psychology (formerly human sociobiology), which appears custom designed to appeal to the evidently deeply ingrained human penchant for reductionistic explanation. Indeed, if we are seeking universals underlying human cognitive function we might do better to look for them in such phenomena as this regrettable reductionist tendency rather than piecemeal in the behaviors themselves. For, despite

Ian Tattersall is a paleoanthropologist at the American Museum of Natural History, Central Park West at 79th Street, New York, NY 10024, where he is a Curator in the Division of Anthropology. His e-mail address is iant@amnh.org. This article is based on a presentation made at the American Association for the Advancement of Science Seminar on Primatology and Human Nature in Washington, D.C., 26-28 January 2001.

[Zygon, vol. 36, no. 4 (December 2001).]

(C) 2001 by the Joint Publication Board of Zygon. ISSN 0591-2385 
the untidiness of most actual human experience, human beings (at least in Western society) appear to prefer simple, all-encompassing explanations of phenomena of whatever kind to the contemplation of the messy, contingent, and often inexplicable facts of human life.

Yet, if we are to take the reductionist route, we have to be willing to blind ourselves to the self-evident complexities of the evolutionary process by which we-like all other living organisms - came to be what we are. And while the ability to remain oblivious to inconvenient facts will presumably always remain a major component of the human behavioral repertoire, if we are ever to make any progress in our understanding of ourselves and of our remarkable underlying cognitive skills, we will ultimately have to accept that both we and the world we live in are more complex than those whose job it is to explain them might wish them to be.

Clearly, the place to start in trying to provide such explanation is with the evolutionary process itself. Mistake how evolution proceeds and the constraints under which this process works, and you are forever condemned to misinterpret its results. It is particularly important to realize this in the present context, since notions of the role of the genes in evolution are heavily dependent on the associated (if rarely articulated) notions of process.

\section{Evolution: A Multilevel Process}

As Niles Eldredge (1979) pointed out years ago, there are two basic themes that must be addressed by any account of evolution that pretends to completeness. One of these is indeed change, at both population and individual levels, in genes and their products. And the other factor, more commonly ignored, is the origination of new species. These two processes not only are conceptually distinct but routinely require the operation of entirely different mechanisms (Tattersall 1994). Yet, particularly in anthropology, the crucial matter of species origins is often considered to be no more than a passive result of morphological change: accumulate enough tiny genetic differences over enough generations, and a new species will inevitably result. The signal of the fossil record, however, indicates that this is not the case. Not only do environments as a whole fluctuate more rapidly than natural selection as traditionally conceived of could be expected to track effectively, but the fossil record shows clearly that, historically, species have tended to come and go rather abruptly rather than displaying gradual changes from one to another over the eons. Nonetheless, since the overwhelming triumph during the years following the end of World War II of the movement known as the Evolutionary Synthesis, which portrayed the process of evolution as little more than gradual shift in population gene frequencies under the guiding hand of natural selection, the tendency in anthropology has been to portray human evolution 
as a linear (or, latterly, as linear as possible) struggle from primitiveness to perfection (Tattersall 1994). The flourishing of this tendency even in the face of rapidly accumulating evidence to the contrary reflects a durable and deeply ingrained anthropological mindset, which those who favor unwieldy names might dub the "transformationist/adaptationist paradigm." For if it were actually true that evolution is simply the result of adaptive gene frequency shifts in populations over long periods of time, then it would follow that we can understand all evolutionary phenomena strictly in terms of adaptation.

Of course, all organisms that have not gone extinct are by definition in some way adapted to their environments. However, when we speak of adaptations we almost invariably refer not to the adaptedness of organisms (or species) as functioning units but to specific attributes of those organisms: to parts of organisms and not the integrated wholes. Equally, the concept of adaptation itself is taken to refer to changes in such specific structures (or behaviors) over time. The tendency thus is to identify unitary attributes and then to study them as if they can be independently tracked through the ages, in isolation from the organisms of which they form part. This is particularly true, it turns out, of behavioral attributes, if only because we already have a useful vocabulary for singling them out. Hence, we talk of the "evolution of bipedality," of the "evolution of cooperation," of the "evolution of the brain," and even of the "evolution of infidelity," while rarely bothering to consider the implications of such broadbrush characterizations. Evolution has become essentially a process of finetuning, over the ages, of myriad separate characters. Yet, the underlying reality is that the "adaptations" thus defined cannot even in concept be independent entities, each with its own evolutionary history that can be pursued at will. For every adaptation is embedded in an individual organism (or, at another level, is the property of a particular taxon), each of which incorporates hundreds or, more likely, thousands of other attributes that could also be regarded as adaptations. And natural selection in the received sense can only vote up or down on individuals (or taxa) as the sum of their parts; it cannot single out particular features to promote or disfavor. Individuals certainly vary in their reproductive success, to which one specific "adaptation" may even make a crucial contribution, positive or negative. At the same time, however, the rest of the genome is inevitably carried along in the same winnowing process, and all of its components will share the same evolutionary fate. In short, if we ignore the phylogenetic histories of the "packages" into which all adaptations are incorporated, we expose ourselves to a severe danger of distorting the evolutionary scenarios we produce.

To avoid this trap, we have to take into account the fact that evolutionary histories are in large part the story of the differential successes of taxa that succeeded or failed in the ecological arena as the overall sums of their 
parts. Of course, nobody would nowadays disagree that natural selection, acting at the level of the local population through the reproductive winnowing of individuals, plays a crucial role in establishing gene frequency changes in those populations over time. Indeed, random sampling aside, there is no other obvious mechanism for producing the genetic and phenotypic diversification of populations within species that provides the basic material of the evolutionary process. But at the same time we have to acknowledge that evolution-that is, whatever it is that produces evolutionary histories-is a complex process in which various levels of action combine to produce the results observed. What is more, we are not speaking here of a hierarchy just of heredity. Certainly, genes, chromosomes, individuals, demes, species, and higher taxa all play their differing parts in a hierarchy of roles. But in producing phylogenetic histories all of these factors act within a conceptually and functionally separate economic hierarchy (individuals, local populations, species, ecological communities, environments). To reduce this complex interaction to little more than gene frequency shifts is to lose sight of the greater part of the story. Novelties arise within individuals and local populations, it is true; but in the longer term new structures and behaviors are triaged not simply within those populations but by competition (with related or unrelated species) and by larger environmental changes (including shifts that are random to a particular population's adaptations).

\section{SELFISH GENES}

Those who call themselves evolutionary psychologists like to claim that the ancestry of their field dates back to Charles Darwin's assertion (1859, 488) that "In the distant future ... psychology will be based on a new foundation, that of the necessary acquirement of each mental power and capacity by gradation." In reality, however, the search for intellectual antecedents for this "discipline" cannot functionally extend back beyond William Hamilton's kin-selection notions of the mid-1960s (Hamilton 1964). These would probably have remained largely unremarked and ungeneralized had they not been publicized a decade later by E. O. Wilson (1975) in his remarkable work Sociobiology: The New Synthesis. However, what really gave evolutionary psychology, as practiced today, its initial impetus was the association of Wilson's ideas on the biological bases of behavior with the mechanisms of the "selfish gene" espoused by Richard Dawkins in his book of that name (Dawkins 1976). In this work Dawkins claimed that the essential targets of evolution by natural selection are not individual organisms, as Darwin had it, and certainly not species as wholes; rather, the basic evolutionary unit is constituted by the genes themselves: the "immortal replicators." Individual organisms are simply transitory vehicles for these replicators, which vie among themselves for evolutionary success. 
Life histories are reduced to little more than the struggle of (by implication helpless) individuals to maximize the number of genes each one contributes to the next generation.

Here, then, is the basis for the notion that individual behaviors are determined by identifiable genes that are sufficiently discrete to be in competition with those for alternative behavioral patterns. In the evolutionary psychological view, the search has become one for "mental adaptations" that have resulted from the action of natural selection during our species' past. Heavily influenced by Darwin's view (nicely enshrined in the quotation above) that the evolutionary process consists of little other than slow, gradual changes in gene frequencies over vast periods of time, many evolutionary psychologists have taken to emphasizing the significance of the "ancestral environment" in which current genotypes allegedly evolved (e.g., Tooby and Cosmides 1990; Irons 1998). Evolution, they believe, takes place too slowly to keep up with rapidly changing environments such as those in which Homo sapiens lives today. To find the "adaptive" causes of specific behaviors exhibited by modern humans, we need not look at the world around us but instead should direct our attention toward the ancestral environment (effectively, that of hunter-gatherers) in which our genes evolved. That this "environment" was as monolithic (in structure or in time) as the choice of term suggests is, of course, debatable: among other considerations, our view is skewed by the fact that the few hunting-gathering societies that survived to form the subject of anthropological study had already been peripheralized to a handful of marginal habitats. Indeed, Dawkins himself (1976) took a distinctly different tack when it came to the analysis of human behaviors. Noting that many behaviors are learned within cultures, Dawkins derived the parallel notion (to genes) of culturally transmitted (and fast-changing) behavioral units that he called "memes." Significantly, this concept has not found favor in mainstream evolutionary psychology.

The problem with all this, of course, is that exactly as in the case of adaptations, genes do not have independent existences. Just as adaptations are embedded in organisms that must succeed or fail as the sums of their parts, genes are embedded in genotypes of staggering complexity that will have greater or lesser reproductive success as functioning wholes. Significantly, these genotypes are passed along through the generations, if not intact, at least with strong associations among their parts. Further, most units (frequently debatable) that we might identify as genes cooperate with others-often many others-in determining particular phenotypes, just as many of the same units influence more than one developmental process in the individual to whom they belong. Thus, while it is fruitless to deny that the behaviors and capacities of organisms are in some way influenced by genetic heritage, it is usually equally unproductive to identify or even to infer genes "for" particular behaviors. Even in those cases where strenuous 
efforts have purported to locate genes for specific human behavioral proclivities (e.g., homosexuality in males), such findings have been equally vigorously contested, and it is in any event usually impossible to know whether the genes involved also play other roles in the development, life, and behaviors of the individual (though they probably do).

\section{The IMPORTANCE OF PHyLOGENy IN RELATION TO BEHAVIOR}

None of this is, of course, to deny the influence of genes, and of the phylogeny they embody, upon individual behaviors. The genotype is, after all, the blueprint upon which each new individual is built, and at some level it certainly determines the range of behaviors possible. The question is simply one of specificity of action, and where evolutionary psychologists are clearly correct is in their perception that species (along with their myriad attributes) are the product of long evolutionary histories. But to equate genes with particular behaviors in the case of an organism as complex cognitively as Homo sapiens is to ask a little much, no matter how closely controlled fight-or-flight responses may be in certain kinds of fish. What genes actually do is guide developmental processes, and in the behavioral arena they specifically guide the development of the controlling organ, the brain.

The incomparably complex human brain is the product of a long phylogenetic history and has evolved through a drawn-out process of intermittent accretions of new structures and differential expansions of preexisting components. Indeed, it is as good an example as exists of the "heterobathmy of synapomorphy" (i.e., the varying time-depths of the various brain components). The resulting "layered" arrangement ensures that our behaviors may be affected by some very ancient structures indeed, as well as by more recent acquisitions; and if we are ever to understand the mysteries of human consciousness, we are going to have to do it in the context of understanding how our cognitive function is influenced by the phylogenetic history of the brain. However, it is very unlikely that we will find that individual behaviors are at all precisely mediated by structures closely tied to separable genetic and/or developmental bases. What is more likely is that behavioral potentials have been increased or modified in the course of primate phylogeny by the enlarging and reorganizing of the brain. Thus, it is probably no coincidence that the primate society showing most similarities to that of humans is said to be that of chimpanzees, also believed by many to be our closest phylogenetic relatives (see Wrangham and Peterson 1996). However, the variation of individual behaviors, both within and among different human and chimpanzee cultures, indicates that such behaviors are not "programmed" in any literal sense.

It is, of course, true that diligent evolutionary psychologists have been able to identify a suite of behavioral "human universals" (e.g., Brown 1991). 
Interestingly, most of these universals (maybe better termed "regularities") have to do with the disparate roles of males and females in human societies. Nobody would contest, of course, that male and female humans tend on average to show significantly different behavioral proclivities that are consistent among diverse cultures. Males do indeed tend to philander, while females tend to bond. Where disagreement occurs is in the explanation of this consistency. Evolutionary psychologists would argue that males and females are "genetically programmed," through the influence of the "ancestral environment," to perform their different roles. But leaving aside the fact that strong variations occur in behavior from individual to individual (and, at another level, from society to society), it is evident that we do not need specific genes to explain the regularities that exist.

Certainly, many biological differences between males and females are genetically determined; but once such determination has been made, it is just as compelling to argue that behavioral differences that may exist are simply by-products of these primary differences. Given that females always have to bear the costs of childbearing and -rearing, while males do not, it is simply a matter of economic advantage that females should exhibit affiliative behaviors toward males, particularly in societies where paternity is recognized. These behaviors may well involve the (variably complete) suppression of the generalized urge to have sex (a species universal, for obvious reasons), something that remains relatively unconstrained among males. As implied earlier, all organisms live in an economic and ecological hierarchy as well as forming part of a genetic one; and, indeed, each individual in his or her lifetime spends immeasurably more time and investment of effort in satisfying economic necessities than in activities that are related to gene propagation. Whether in any particular case strong regularities in behavior result purely from calculations by each individual is, of course, debatable; if the economic advantage to a given behavior is significant, behavioral traits will clearly be readily reinforced by cultural tradition.

\section{BEHAVIORAL INNOVATION IN HUMAN EVOLUTION}

The fossil and archaeological records of the human family indicate that hominid phylogeny over the past 4 to 5 million years (myr) has in general conformed to the overall pattern typical for living things. Homo sapiens is but one terminal twig on a large branching bush of species that already number twenty, with more to come. About 1.8 myr ago, in Kenya, we have evidence that four hominid species shared not only the same continent but the same landscape. What is truly remarkable, in fact, is that our species is the lone hominid in the world today (Tattersall 2000). This by itself suggests that there is something unusual about us, which should probably make us resistant to broad generalizations based on our antecedents. 
It is worth briefly recapping the history of hominid innovation with the aim of asking what this something is.

For the first 2 to 3 myr of hominid history, the record contains fossils only of australopiths, small-bodied early bipeds with small brains and large faces, that retained many features that would have been useful for life in the trees. This bipedal-but-arboreal habitus was a very successful one, remaining essentially unchanged for a long time, even as numerous distinct australopith species came and went. Although no australopith is known for certain to have made stone tools, it seems likely that the first stone tool maker was something we would call an australopith: after all, any technological innovator has to belong to a preexisting species and cannot differ too much from its nontechnological parents. The earliest stone tools come from sites in eastern Africa, date from about 2.5 myr ago, and consist of simple sharp flakes knocked off small cobbles. At about the same time we begin to find fragmentary fossils often assigned to "early Homo." However, the first humans of demonstrably human body build (though with smallish brains) do not turn up until about 1.8 myr ago, though this is considerably before the next innovation in stone tool making (the Acheulean handaxe, consciously shaped to a specific form), some 1.6 myr ago. At around the earlier date, hominids exited Africa for the first time, giving rise to new species in Asia, Europe, and probably elsewhere too, but not spawning any demonstrably new technology until about 400,000 years ago, when the first strong evidence for hearths and constructed shelters (and the first example of wooden throwing spears) shows up. About 100,000 years thereafter, the "prepared-core" stone tool makes its appearance, wherein a stone core was carefully shaped until a single blow would detach a more or less finished tool. The best-known practitioners of this technology were the Neanderthals, a distinctive, large-brained hominid species that flourished in Europe and western Asia in the period between about 200,000 and 30,000 years ago.

The Neanderthals were accomplished in many ways and famously buried their dead, at least occasionally; but the behavioral record they left behind was totally eclipsed by that of the first Homo sapiens who began entering Europe about 40,000 years ago. Up until the appearance of modern humans, it is not unfair to say that throughout hominid history new kinds of humans had mostly done what their predecessors had done, if a little better (Tattersall 1998). The technological record is one of extremely sporadic accretion rather than revolution. With Homo sapiens (or at least behaviorally modern Homo sapiens), the break with precedent was dramatic, with the first appearances, virtually simultaneously, of cave art, engraving, carving, notation, music, elaborate burial of the dead, body ornamentation, technological acceleration, and a host of the other attributes that we call our own-including intolerance of related species, for within 
a small span of millennia, the formerly ubiquitous Neanderthals were gone forever.

What happened? It seems that something extraordinary and unprecedented occurred with the emergence of behaviorally modern Homo sapiens (behaviorally archaic Homo sapiens were around almost 100,000 years ago but did not make much of a splash). Judging from the (admittedly biased) record they left behind in Europe, the crucial difference between the former and all earlier hominids was the appearance of symbolic thought. Apparently by this stage in human evolution, the human brain had acquired the potential to produce an entirely novel cognitive structure, which, in the absence of any evidence of biological change, must have been released by some cultural innovation. What that innovation was is uncertain, but it may have involved the invention of language, the quintessentially symbolic activity (and for which the human vocal tract was already prepared; see Tattersall 1998). The emergence of modern human cognition was also the culmination of a long process of brain evolution in the human lineage, for it clearly did not come out of nowhere. The resulting layered structure of our brains has important behavioral consequences, particularly in the interaction between the symbolic and emotional components of our consciousness (if, indeed, this crude distinction is permissible, as at some level it probably is).

If all or most of these conjectures are true, Homo sapiens is not simply an improvement on what went before, though we certainly did not leave our past entirely behind. As a cognitive and behavioral entity, our species is truly unprecedented. Our consciousness is an emergent quality, not the result of eons of fine-tuning of a single instrument. And, if so, it is to this recently acquired quality of uniqueness, not to hypothetical "ancestral environments," that we must look in the effort to understand our often unfathomable behaviors. We and we alone, as individuals, are responsible for what we are.

\section{NOTE}

I would like to thank Audrey Chapman, Jim Miller, and Bob Sussman for having inspired and organized the seminar for which these thoughts were prepared, and all of the participants for the stimulating and collegial atmosphere they created. I would particularly like to thank my discussant, Wentzel van Huyssteen, for his thoughtful comments.

\section{REFERENCES}

Brown, D. E. 1991. Human Universals. New York: McGraw Hill.

Darwin, Charles R. 1859. On the Origin of Species. London: John Murray.

Dawkins, Richard. 1976. The Selfish Gene. Oxford: Oxford Univ. Press.

Eldredge, Niles. 1979. "Alternative Approaches to Evolutionary Theory." Bulletin of the Carnegie Museum of Natural History 13:7-19.

Hamilton, William. 1964. "The Genetical Evolution of Social Behaviour," parts 1 and 2. Journal of Theoretical Biology 7:1-52. 
Irons, William. 1998. "Adaptively Relevant Environments versus the Environment of Evolutionary Adaptiveness." Evolutionary Anthropology 6:194-204.

Tattersall, Ian. 1994. "How Does Evolution Work?” Evolutionary Anthropology 3:2-3. 1998. Becoming Human: Evolution and Human Uniqueness. New York: Harcourt

Brace. 2000 . “Once We Were Not Alone.” Scientific American 282:56-62.

Tooby, John, and Leda Cosmides. 1990. "The Past Explains the Present: Emotional Adaptations and the Structure of Ancestral Environments." Ethology and Sociobiology 11:375424.

Wilson, E. O. 1975. Sociobiology: The New Synthesis. Cambridge: Harvard Univ. Press.

Wrangham, Richard, and Dale Peterson. 1996. Demonic Males: Apes and the Origins of Human Violence. New York: Houghton Mifflin.

Wright, Robert. 1998. The Moral Animal. New York: Pantheon. 